\title{
PITX2 Overexpression Leads to Atrial Electrical Remodeling Linked to Atrial Fibrillation
}

\author{
Jieyun Bai ${ }^{1}$, Yaosheng $\mathrm{Lu}^{1}$, Andy C Y Lo ${ }^{2}$, Jichao Zhao ${ }^{2}$ \\ ${ }^{1}$ Department of Electronic Engineering, College of Information Science and Technology, Jinan \\ University, Guangzhou, China \\ ${ }^{2}$ Auckland Bioengineering Institute, The University of Auckland, Auckland, New Zealand
}

\begin{abstract}
Although functional analysis has shown that electrical remodelling in Atrial fibrillation $(A F)$ patients is related to PITX2 overexpression, this link between PITX2 overexpression and the likelihood of atrial arrhythmias remains to be demonstrated directly. Therefore, utilizing computer modelling, this study was to quantify the proarrhythmogenic effects of PITX2 overexpression in the human atrium at cell and tissue levels. In simulations, Courtemanche et al.'s model of human atrial cell AP was modified to incorporate experimental data on changes of $I_{K s}$ and $I_{C a L}$ induced by PITX2 overexpression. The cell models for normal and AF type ionic currents $\left(I_{K s}\right.$ and $\left.I_{C a L}\right)$ were incorporated into the homogeneous multicellular $2 D$ tissue model. Effects of electrical remodelling induced by PITX2 overexpression were quantified on ionic currents, $A P$ profile and AP duration (APD). Dynamic behaviors of re-entrant excitation waves in the $2 D$ model were characterized. It was shown that the PITX2 overexpression abbreviated atrial APD. In the 2D model, the PITX2 overexpression also stabilized and accelerated re-entrant excitation waves, leading to rapid and sustained re-entry. In conclusion, increased $I_{K s}$ and reduced $I_{C a L}$ due to the PITX2 overexpression increases atrial susceptibility to arrhythmia due to increased tissue vulnerability, shortened APD and abbreviated wavelength.
\end{abstract}

\section{Introduction}

Atrial fibrillation (AF) is an extremely common clinical problem, with an incidence that is rising, and significantly affects morbidity and mortality[1]. Although current therapeutic methods, which include antiarrhythmic drug treatment, cardiac ablation and surgical therapy, have been significantly improved over the past century, their efficacy remains suboptimal and adverse effects exists[2]. These adverse effects (AF-induced remodelling) are thought to promote AF which is characterized a fast and irregular heart beat[3]. However, genome-wide association studies (GWAS) have identified a genetic predisposition underlying AF.

Human AF GWAS have implicated multiple transcription factors, including the paired like homeodomain transcription factor 2 (PITX2). Three PITX2 isoforms (PITX2a, PITX2b and PITX2c) are highly expressed in humans during development. PITX2c is the predominant cardiac isoform[4]. Dysfunction of PITX2c predisposes to AF associated with both decreased and increased expression (or activity) of PITX2c[5]. In a previous study, Pérez-Hernández et al. found that PITX2c mRNA expression was significantly higher in human atrial myocytes from chronic AF patients and this increase correlated with the $I_{K s}$ increase and $I_{C a L}$ decrease[6]. Although these changes contributed to electrical remodelling, the mechanisms underlying chronic AF was not demonstrated directly.

Since complex electrical wave dynamics observed during chronic AF is determined by the action potential (AP) profile and AP duration. Here, using a multi-scale computational model of human atrial tissues, we quantified the impact of PITX2c overexpression on atrial electrical activity at the cellular and two-dimensional (2D) tissue levels.

\section{Methods}

The well-known Courtemanche-Ramirez-Nattel (CRN) model[7] of the human atrial myocytes was chosen to investigate the proarrhythmic effects of the up-regulated PITX2c, because this model was wildly used to study spatiotemporal characteristics of spiral waves at the tissue and organ levels. These multicellular models are given by

$$
C_{m} \frac{d V_{m}}{d t}=-I_{\text {ion }}+\nabla \cdot\left(D \nabla V_{m}\right)
$$

where the diffusion coefficient $(D)$ is set to $0.031 \mathrm{~mm}^{2} / \mathrm{ms}$ which can lead to a plane wave with a conduction velocity (CV) of $0.27 \mathrm{~mm} / \mathrm{ms}$. $V_{m}$ denotes the transmembrane potential, $C_{m}$ denotes the total membrane capacitance, the sum of all ionic currents $\left(I_{i o n}\right)$ is given by

$$
\begin{aligned}
I_{\text {ion }}= & I_{N a}+I_{C a L}+I_{N a b}+I_{C a b}+I_{p C a}+I_{K u r} \\
& +I_{N C X}+I_{N a K}+I_{t o}+I_{K r}+I_{K s}
\end{aligned}
$$


where $I_{C a L}$ is reduced by $50 \%$ and $I_{K S}$ is increased by twofold under chronic AF condition, compared with the normal condition (Figure 1A).

The CRN model for human atrial myoctes

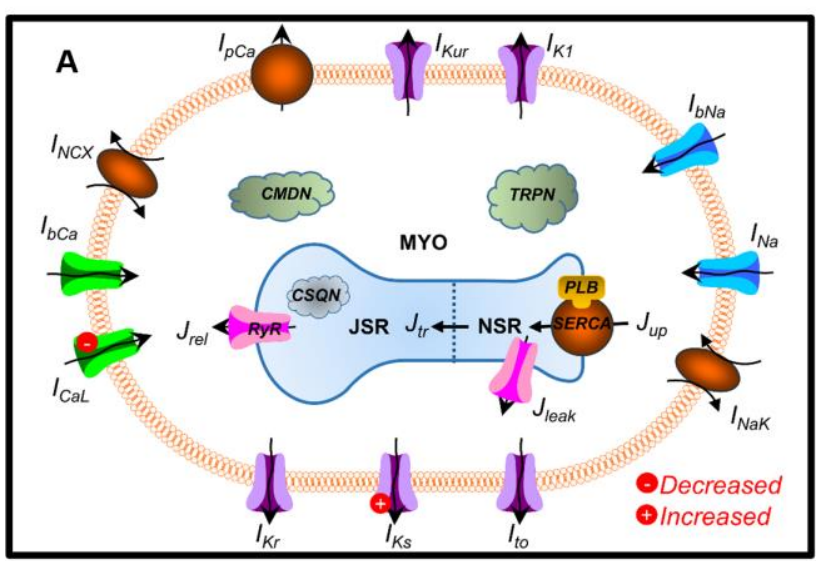

The multicellular model with $375 \times 375$ nodes

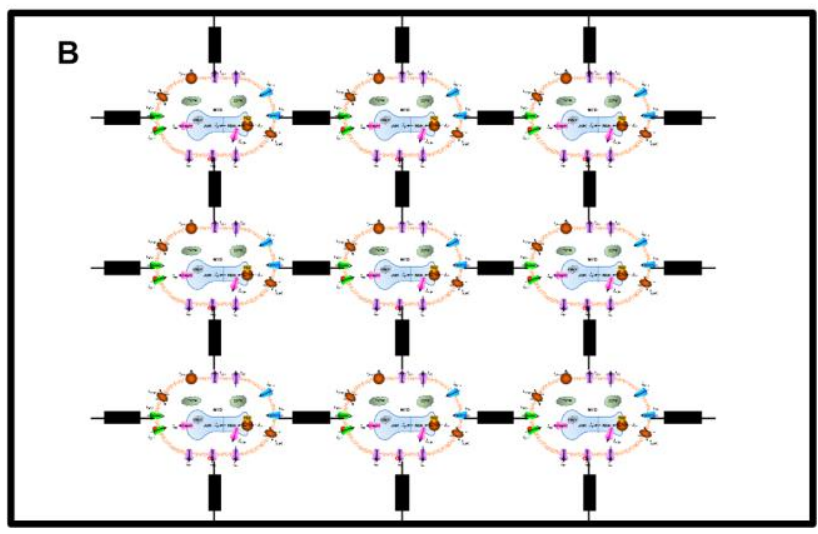

Figure 1. Schematic diagrams for human atrial myocytes and homogeneous multicellular tissues. (A) Under chronic AF condition, $I_{K s}$ is increased and $I_{C a L}$ is reduced. (B) Human atrial cell models were connected with gap junctions to generate the multicellular tissue models. Here, a homogeneous tissue model with $375 \times 375$ nodes.

In this study, we designed a 2D sheet model with $375 \times 375$ nodes to investigate the temporal-spatial characteristics of electrical waves due to PITX2c overexpression. The standard S1-S2 protocol was used to induce spiral waves. A plane S1 stimulus was applied to the bottom boundary to initiate a plane wave. Once this wave had passed over the first half of the domain, an S2 stimulus was applied to the bottom left quarter of the domain to initiate a spiral wave.

The partial differential equations were solved by an explicit forward Euler approximation. Time and space steps were set to be $0.005 \mathrm{~ms}$ and $0.1 \mathrm{~mm}$, respectively. The strength and duration of stimuli were set to be -80 $\mathrm{pA} / \mathrm{pF}$ and $0.5 \mathrm{~ms}$, respectively. $\mathrm{APD}_{90}$ was defined as $\mathrm{AP}$ duration (APD) at $90 \%$ repolarization.

\section{Results}
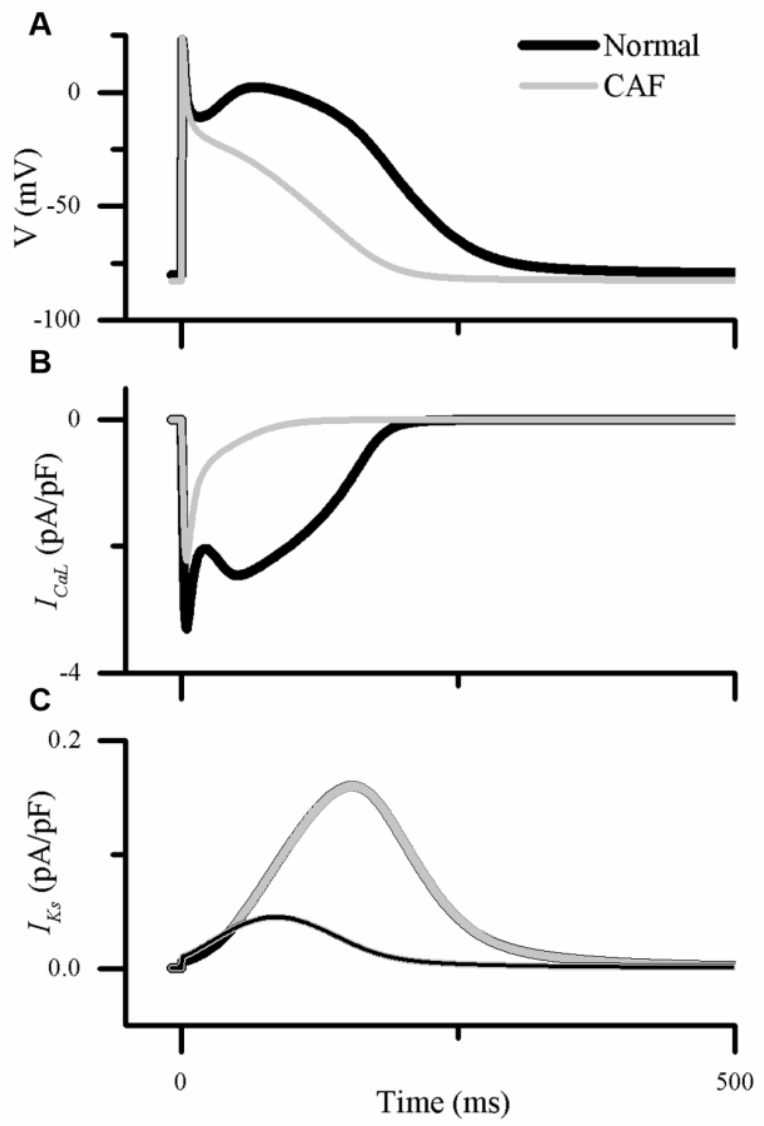

Figure 2. $\mathrm{AP}, I_{C a L}$ and $I_{K s}$ under normal and $\mathrm{CAF}$ conditions. (A) The increase of PITX2c causes APD shortening and a spike-type AP. (B) Compared with the normal condition, the $I_{C a L}$ amplitude is reduced and the dome of $I_{C a L}$ was eliminated under the CAF condition. (C) The outward potassium current $I_{K s}$ was significantly increased during the phase 3 of AP under the CAF condition.

As described in Figure 2, PITX2c overexpression led to APD shortening by reducing $I_{C a L}$ and increasing $I_{K s}$. The dome of AP under the normal condition was eliminated under the CAF condition. The measured $\mathrm{APD}_{90}$ was decreased from $267 \mathrm{~ms}$ to $172 \mathrm{~ms}$ (Figure 2A). During the repolarization period of $\mathrm{AP}$, a spike-type $I_{C a L}$ during the phase 2 (Figure 2B) and a dome-type $I_{K s}$ during the phase 3 (Figure 2C) were observed under the CAF condition. Compared with the normal condition, changes in AP can be attributed to reduced $I_{C a L}$ and increased $I_{K s}$. 


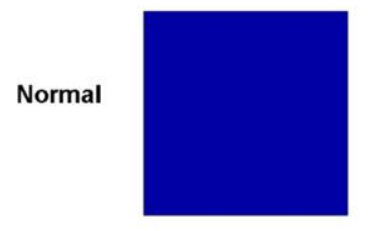

$0 \mathrm{~ms}$

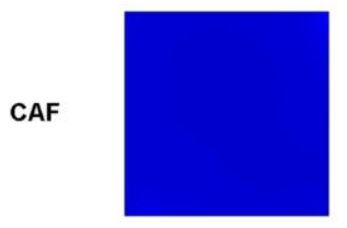

$0 \mathrm{~ms}$

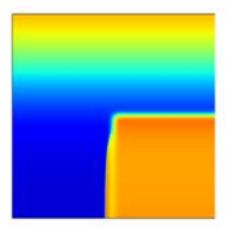

$390 \mathrm{~ms}$

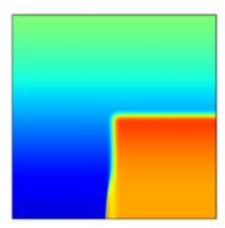

$270 \mathrm{~ms}$

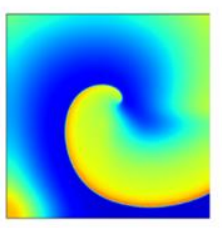

$600 \mathrm{~ms}$

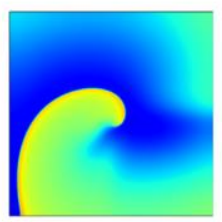

$600 \mathrm{~ms}$

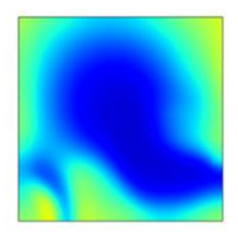

$800 \mathrm{~ms}$

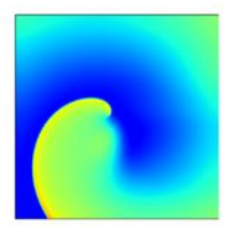

$800 \mathrm{~ms}$

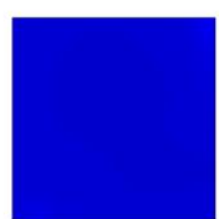

$1000 \mathrm{~ms}$

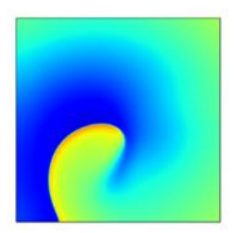

$1000 \mathrm{~ms}$

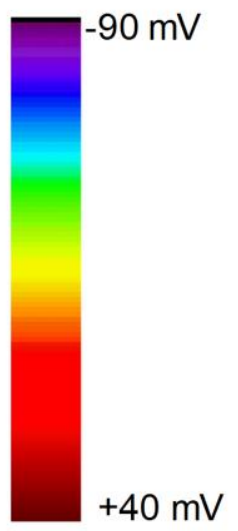

$25 \mathrm{~mm}$

Figure 3. Simulated spiral waves in a 2D model of human atrium. Top panels show results from $2 \mathrm{D}$ re-entrant wave simulation under normal condition. Bottom panels show results from the CAF condition. Frames from the 2D simulation at time $=0,270,600,800$ and $1000 \mathrm{~ms}$ are shown.

To further examine whether the PITX2c overexpression promotes and perpetuates AF, its effects on the temporalspatial dynamics of spiral waves were investigated. Figure 3 shows the increase in PITX2c under the CAF condition supported a spiral wave to sustain. After the $\mathrm{S} 1$ stimulus, the bottom half domain recovered at $390 \mathrm{~ms}$ under the normal condition and $270 \mathrm{~ms}$ under the CAF condition. The life span of a spiral wave was $<900 \mathrm{~ms}$ and $>1000 \mathrm{~ms}$, in the normal and CAF conditions, respectively. Thus, early repolarization and sustained spiral wave under the CAF condition can be attributed to wavelength abbreviation due to APD shortening.

\section{Discussion}

This study investigated effects of electrical remodelling due to PITX2c overexpression under the CAF condition on atrial electrical activity at the cellular and tissue levels. Our main findings are as follows: (1) The electrical remodelling arising from the up-regulated PITX2c abbreviated APD, and (2) it stabilized and allowed electrical waves to maintain in smaller tissue sizes that could not be occurred under the normal condition. Consequently, these findings demonstrate that PITX2c overexpression increases the likelihood of AF due to APD shortening and thereby wavelength abbreviation, which facilitate genesis and maintenance of re-entrant excitation waves.

APD shortening is pro-arrhythmic. AF is characterized by a marked shortening of the atrial APD and effective refractoriness period as a consequence of alterations in the expression and function of $I_{C a L}$ and $I_{K s}$. In CAF patients, the $I_{C a L}$ decrease and $I_{K s}$ increase critically contribute to the
APD shortening[8-10]. In our study, under the CAF condition, APD shortening and spiral waves can be attributed to the $I_{C a L}$ decrease and $I_{K s}$ increase due to the PITX2c overexpression.

\section{Conclusion}

Increased $I_{K s}$ and reduced $I_{C a L}$ due to the PITX2c overexpression increases atrial susceptibility to arrhythmia due to increased tissue vulnerability, shortened APD and abbreviated wavelength, which, in combination, facilitate initiation and maintenance of re-entrant excitation waves.

\section{Acknowledgements}

This work was supported by he National Natural Science Foundation of China (No. 61901192) (J.B.) and the Science and Technology Planning Project of Guangdong Province (No.2015B020214004 and No.2015B020233010) (Y.L.).

\section{References}

1. Heijman, J., et al., Translational challenges in atrial fibrillation. Circulation Research, 2018. 122(5): p. 752-773.

2. Hansen, B.J., et al., Atrial fibrillation driven by micro-anatomic intramural re-entry revealed by simultaneous sub-epicardial and sub-endocardial 
optical mapping in explanted human hearts. European heart journal, 2015. 36(35): p. 23902401.

3. Colman, M.A., et al., Pro - arrhythmogenic effects of atrial fibrillation - induced electrical remodelling: insights from the three dimensional virtual human atria. The Journal of physiology, 2013. 591(17): p. 4249-4272.

4. Syeda, F., P. Kirchhof, and L. Fabritz, PITX2dependent gene regulation in atrial fibrillation and rhythm control. The Journal of Physiology, 2017. 595(12): p. 4019-4026.

5. Bai, J., et al., Ionic and cellular mechanisms underlying TBX5/PITX2 insufficiency-induced atrial fibrillation: Insights from mathematical models of human atrial cells. Scientific reports, 2018. 8(1): p. 15642.

6. Pérez-Hernández, M., et al., Pitx2c increases in atrial myocytes from chronic atrial fibrillation patients enhancing I Ks and decreasing I Ca, L. Cardiovascular research, 2015. 109(3): p. 431441.

7. Courtemanche, M., R.J. Ramirez, and S. Nattel,
Ionic mechanisms underlying human atrial action potential properties: insights from a mathematical model. American Journal of Physiology-Heart and Circulatory Physiology, 1998. 275(1): p. H301-H321.

8. González de la Fuente, M., et al., Chronic atrial fibrillation up-regulates $\beta 1$-adrenoceptors affecting repolarizing currents and action potential duration. Cardiovascular research, 2012. 97(2): p. 379-388.

9. Barana, A., et al., Chronic atrial fibrillation increases microRNA-21 in human atrial myocytes decreasing L-type calcium current. Circulation: Arrhythmia and Electrophysiology, 2014. 7(5): p. 861-868.

10. Caballero, R., et al., In humans, chronic atrial fibrillation decreases the transient outward current and ultrarapid component of the delayed rectifier current differentially on each atria and increases the slow component of the delayed rectifier current in both. Journal of the American College of Cardiology, 2010. 55(21): p. 23462354.

\author{
Address for correspondence: \\ Dr. Jieyun Bai \\ Department of Electronic Engineering \\ College of Information Science and Technology \\ Jinan University, Guangzhou, China \\ E-mail: bai_jieyun@126.com
}

